\title{
Tiny Footprints Embedded on Grieving Mother's Heart: Stillbirths in the Health and Demographic Surveillance System
}

\author{
Muhammad llyas*, Murtaza Ali, Muhammad Imran Nisar, Fyezah Jehan and Abdul Momin \\ Kazi
}

Pediatrics, The Aga Khan University, Karachi, Karachi, Pakistan

\author{
Objective \\ Objective: To describe characteristic of stillbirth in a diverse \\ population in Karachi health and demographic surveillance system.
}

\section{Introduction}

Stillbirth is an unfortunate event in a woman life which remains uncounted in developing countries, thus, seldom caught attention until recently. Among 3.2 million stillbirths globally, 98\% occurs in LMICs with majority in South Asia, and $75 \%$ of those are preventable. Globally, it counts as equal to neonatal deaths and is not mentioned in MGDs, global charters and programs priority. Besides immense information gap, it is mostly not part of vital registration system. Mostly, the data for stillbirths is mostly collected in demographic surveys, clinical studies or retrospective records, underestimating the counts. Besides, lack of optimal national vital registration system, Pakistan has highest rate of stillbirth. Hence, to collect prospective data, efforts are made by Department of Paediatrics of Aga Khan University to maintain a Demographic \& Health Surveillance System (DHSS) at Karachi to provide more robust data over years.

\section{Methods}

Our catchment area is $19 \mathrm{sq} \mathrm{km}$ with a population of around 274,856 with 69699 females between 15 to 49 years of age. Surveillance catchment area is divided into clusters/blocks of about 200 to 250 structures, each marked with a unique number. Each married woman, pregnant woman and $<5$ year child's unique ID is collated with GPS coordinate of structures they live in. Block boundary is mapped using GPS track log technique. Structures, streets and landmarks are digitized through GIS. Our community health workers (CHWs) visit each household quarterly in DHSS area to identify new pregnancies and follow pregnant woman until pregnancy outcome. Further verbal autopsies (VA) are conducted for all stillbirths, $<5$ years children deaths and adult female deaths (13-49 years) including maternal deaths to obtain cause-specific mortality. VA's are attempted after allowing 2 weeks of grieving time to family.

\section{Results}

During 2012 we captured 13979 pregnant women, 9136 live births and 239 still births yielding stillbirth rate of 25.5 per 1000 births. VAs for $79 \%$ stillbirths was completed. In $82 \%$ of VA interview respondent were mothers. Majority stillbirths were Males $(n=107) 58 \%$. Most number of stillbirths occurred in hospitals $47.5 \%$ and $43 \%$ occurred in multi gravida women. $33 \%$ pregnant women with SB reported high blood pressure, $2.1 \%$ had epilepsy during pregnancy and $2.7 \%$ had convulsion during last month of pregnancy. Furthermore $6.6 \%$ of the women with SB had excessive vaginal bleeding during first six month of pregnancy, $9.3 \%$ during last month of pregnancy, and nearly $25 \%$ during the intrapartum period. $72 \%$ of SBs were delivered by doctors, nurses or midwives and $28 \%$ by tradition birth attendants (TBAs). $88 \%$ women had normal vaginal deliveries, $12 \%$ had caesarian section; $22.4 \%$ were premature and $89 \%$ were fresh stillbirths.

\section{Conclusions}

Majority of still births occur near to birth and can be prevented by improving the quality of obstetric care especially around the time of birth.

\section{Keywords}

Stillbirths; Demographic \& Health Surveillance System; Verbal Autopsy; Pakistan

\section{References}

Ilyas, M., Nisar, M. I., Kazi, A. M., Ali, M. T., \& Zaidi, A. (2014). A Health and Demographic Surveillance System in a Low Socioeconomic Setting in Karachi, Pakistan. Online Journal of Public Health Informatics, 6(1).

Ali, M. T., Kazi, A. M., Nisar, M. I., Ilyas, M., \& Zaidi, A. (2014). Geospatial Reporting of Health Demographic Surveillance in a Peri Urban Setting. Online Journal of Public Health Informatics, 6(1).

Stillbirth Collaborative Research Network Writing Group. Causes of death among stillbirths. JAMA: the journal of the American Medical Association306.22 (2011): 2459.

Lawn, Joy E., et al. Stillbirths: Where? When? Why? How to make the data count? The Lancet 377.9775 (2011): 1448-1463.

*Muhammad llyas

E-mail: ilyas.muhammad@aku.edu 